\title{
Algorithm for Computer Chess
}

\author{
PAUL T E CUSACK*
}

Independent Researcher, BSc E, DULE, 1641 Sandy Point Rd, Saint John, NB, Canada E2K 5E8, Canada

*Corresponding Author: PAUL T E CUSACK, Independent Researcher, BSc E, DULE, 1641 Sandy Point Rd, Saint John, NB, Canada E2K 5E8, Canada

\begin{abstract}
This paper provides a foundation upon which to build a chess program. The game of chess models the universe, so AT Math applies.
\end{abstract}

Keywords: Programming Chess; Chess Algorithm; Game of Life Physics; AT Math.

\section{INTRODUCTION}

Chess was invented in either India or China. I suppose that is hotly debated. Whomever invented chess, knew a lot more about mathematical physics than we thought. I am a novice player myself, or at least I used to be. I owned a computer chess game that neither myself nor any of my friends could beat on level 1. There were 16 levels! In this paper, I provide a chess algorithm based on AT Math and what we know of the Game of Life Physics. The chess board is a model of the universe. So let's being the programming of a chess computer.

Good Move=1/Bad Move

Good Move $=\sin \theta$ from $0-\pi$

Neutral Move $=$ Good $=1 / \mathrm{Bad}$

$\sin \theta=1 / \sin \theta$

$\sin ^{2} \theta=1$

$\sin \theta= \pm 1$

Good move $=\sin \theta=1$

$\theta=\pi / 2$

Bad move $=\sin \theta=-1$

$\theta=3 \pi / 2$

Neutral move $=\sin \theta=0$

$\theta=\pi$

$3 \pi / 2 \leq \pi \leq \pi / 2$

The first 14 moves $(2 \times 7)$ of a chess match are usually neutral moves. $S^{\prime}=2 t-1=0$

$2 \mathrm{t}-1=0$

$2(14)-1=27=\mathrm{c}^{3}$

The Mind $\mathrm{L}=\mathrm{Ln} \mathrm{t}+\mathrm{c}^{3}$

$=\mathrm{M}+2 \mathrm{t}-1$ where $\mathrm{t}=14$

$\mathrm{L}=\mathrm{Ln} \mathrm{t}+2(14)-1$

$-25=\operatorname{Ln} \mathrm{t}$ 
$25=-\operatorname{Ln} t$

$\mathrm{t}=07.200$

$\mathrm{S}=\mathrm{E}-\mathrm{M}$

$1 / 72=(-18)+1 / 9)$

$\mathrm{t}^{2}-\mathrm{t}-1=1 / 7.2$

$\mathrm{t}^{2}-\mathrm{t}-113.88=0$

$\mathrm{t}=1678 \sim 1 / 6$

$\mathrm{t}=678 \mathrm{E}=147.4$

$\mathrm{M}=\mathrm{Ln} \mathrm{t}=6=403=\mathrm{Re}=\mathrm{t}$

7 Good Moves $=\mathrm{E}=(1-\operatorname{Ln} \mathrm{t})^{7}$

$7^{1 / 7}=(1-\operatorname{Ln} \mathrm{t})$

$1.320=1-\operatorname{Ln} \mathrm{t}$

$0.320=-\operatorname{Ln} t$

Ln $\mathrm{t}=0.320=-137.7$

$\mathrm{t}^{2}-\mathrm{t}-1=0$

$E=-0.4795$

$\mathrm{t}=1 / \mathrm{E}=1 / 0.48=-1 / 2.08 \sim-1 / 2$

$3(-1 / 2)-1=-2=\mathrm{L}$ mind

The Mind

$\mathrm{L}=\mathrm{Ln} \mathrm{t}+\mathrm{c}^{3}$

$=4+27$

$=31=12$ th Prime Number

$\operatorname{Lim} x \rightarrow \infty 12 /\{31 / \operatorname{Ln} 31]=1$

$1329=1$

$1 / 1329=752$

$1 / \mathrm{s}=752=\mathrm{Ln} \mathrm{s}$

$\mathrm{s}=118.6=\mathrm{M}_{\mathrm{T}}$

$1 / \mathrm{s}=0.8433$

$=\sin 57.49^{\circ}$

$\sim \sin 1$

$=\sin \mathrm{E}=1 / \mathrm{s}$

$\mathrm{s}=\mathrm{csc} \mathrm{E}=\mathrm{M}$

$\mathrm{s}=|\mathrm{E}| \mathrm{t} \mid \sin \theta$

$1 / \mathrm{s}=1 / \sin \mathrm{E}=(1)(1) \sin \theta$

$1=\sin ^{2} \theta$

Euler's Identity:

$\sin ^{2} \theta+\cos ^{2} \theta=1$

$1=\cos ^{2} \theta=1$ 
$\cos \theta=1$

$\theta=0^{\circ}, 2 \pi$

$1 / \cos \theta=\sin \theta$

$1=\sin ^{2} \theta$

$\sin \theta= \pm 1$

$\sin \theta=1$

$\theta=\pi / 2$

$\sin \theta=-1$

$\theta=3 \pi / 2$

Energy:

$1 / \sin \mathrm{E}=-1 \quad \mathrm{E}=3 \pi / 2=33 \quad 33 / 11=3$

$1 / \sin \mathrm{E}=0 \quad \mathrm{E}=0, \pi, 2 \pi=22 \quad 22 / 11=2$

$1 / \sin \mathrm{E}=1 \mathrm{E}=\pi / 2=11 \quad 11 / 11=1$

$\mathrm{t}=3 ; 2 ; 1 \Rightarrow$ Game of Life Physics

$\mathrm{T}+\mathrm{T}+\mathrm{T}+\mathrm{T}+\mathrm{T}+\mathrm{T}+\mathrm{T}=7$

$\mathrm{F}+\mathrm{F}+\mathrm{F}+\mathrm{F}+\mathrm{F}+\mathrm{F}+\mathrm{F}=0$

So the range of moves yields Energy from 0-7

$\mathrm{E}=(1-\operatorname{Ln} \mathrm{t})^{7}$

$\mathrm{t}=1 ; 2 ; 3$ [Game of Life Physics]

$\mathrm{E}_{1}=1 / 1=(1-\operatorname{Ln} \mathrm{t})^{7}$

$\mathrm{t}=1$

$\mathrm{E}_{2}=(1-\operatorname{Ln} \mathrm{t})^{7}$

$1 / 2^{1 / 7}=(1-\operatorname{Ln} \mathrm{t})$

$0.906-1=-\operatorname{Ln~t}$

$\mathrm{t}=1.0998$

$\mathrm{E}_{3}=(1-\operatorname{Ln} \mathrm{t})^{7}$

$1 / 3^{1 / 7}=(1-\operatorname{Ln} \mathrm{t})$

$\mathrm{t}=11.56=1 / \sin 60$

$\mathrm{E}=(1-\operatorname{Ln} \mathrm{t})^{7}$

$0.4935^{1 / 7}=(1-\operatorname{Ln} \mathrm{t})$

125.6=1-Ln $\mathrm{t}$

$\mathrm{t}=1.2919$

$1.2919^{2}=1.2919-1=6.228=1 / 1605 \sim 1 / 1602=1 /$ electrical charge

$=1 / 16=2^{7} / 2^{11}$ ( 7 moves ahead out of the 11 dimension universe. $)$

To program the computer, there must be an interaction between the pieces and board.

Take a piece $=+1$

Loose a piece $=-1$

Neither Take nor loose $=0$ 
$=\mathrm{TNL}$

8 Pawn $2 \times \pi / 2=\pi=3.14 \times 8=251$

2 Rook $4=2 \pi=6.28 \times 2=125.6$

2 Knight $4=2 \pi$

2Bishop $4=2 \pi$

1 King $8=4 \pi=1.256 \times 1$

1Queen $8=4 \pi=125.6 \times 1$

$\Sigma=257.3$

PVal. $\cdot \pi / 2=404=\operatorname{Re}$

$\rho /(1 \operatorname{rad} x \operatorname{Re})$

$=127.3 / 404=3.15 \sim \pi$

$\Pi=2 \times 257.3=514.6$

$\mathrm{t}^{2}-\mathrm{t}-1=0.5146$

$\mathrm{t}=137.4 ; 374=1 / 267=1 / \mathrm{SF}$

$\mathrm{M}=\mathrm{Ln} \mathrm{t}=514=167 \Rightarrow$ Cosmic Pyramid $\Rightarrow \mathrm{SE}=\mathrm{SE}$ '

The Universe has Entropy $\Delta \mathrm{S}=0$

God and Man are opposite of Entropy always working against the universe.

$\mathrm{dS} / \mathrm{dt}=0=\mathrm{SE}=2 \mathrm{t}-1$

$\int \mathrm{dS} / \mathrm{dt}=\mathrm{S}=\int(2 \mathrm{t}-1)$

$=\mathrm{t}^{2}-\mathrm{t}-1$

$\Delta \mathrm{S}=\mathrm{Q} / \mathrm{T}=\mathrm{Q} / 273.15 \mathrm{~K}=0$

$\mathrm{Q}=0$ Adiabatic Process.

$2 \mathrm{t}-1=\mathrm{Q}$

$\mathrm{t}=1 / 2$

$\Delta \mathrm{S}=\mathrm{S}_{\mathrm{f}}-\mathrm{S}_{\mathrm{i}}=0=\Delta \mathrm{S}=\mathrm{Q} / \mathrm{T}=0 / \mathrm{T}=0$

$0=\Delta \mathrm{S} / \Delta \mathrm{t}=\mathrm{dS} / \mathrm{dt}=2 \mathrm{t}-1$

Integrate:

$\mathrm{S}=\mathrm{t}^{2}-\mathrm{t}-1$

$\mathrm{Q} / \mathrm{R}=\mathrm{t}^{2}-\mathrm{t}-1$

273.15 Q/T=273.15 $\mathrm{t}^{2}-273.15 \mathrm{t}-273.15$

$Q=273 t^{2}-273 t-273$

$\mathrm{Q}=[1+\sqrt{3} \sqrt{ }]\left[\mathrm{t}^{2}-\mathrm{t}-1\right]$

$\mathrm{Q}=\mathrm{t}^{2}-\mathrm{t}-1-\sqrt{3} \mathrm{t}^{2}-\sqrt{3}-\sqrt{3}$

$\mathrm{Q} / 732=732 \mathrm{t}^{2}-732 \mathrm{t}-\sqrt{3}$

$0=\mathrm{t}^{2}-\mathrm{t}-0.002366-\mathrm{Q} / 273$

Approximates to:

$\mathrm{t}^{2}-\mathrm{t}=0$

$\mathrm{t}(\mathrm{t}-1)=0$

$\mathrm{t}=0 ; 1$ 
$\mathrm{t}^{2}-\mathrm{t}-1=0$

$0^{2}-0-1=-1$

$1^{2}-1-1=-1$

Therefore $\mathrm{E}=-1$

$\mathrm{E}=(1-\operatorname{Ln} \mathrm{t})^{7}$

$-1=(1-\operatorname{Ln} \mathrm{t})$

$-1-1=-\operatorname{Ln} \mathrm{t}$

$2=\operatorname{Ln} \mathrm{t}$

$\mathrm{t}=7389 \mathrm{E}=1353$

So $\mathrm{t}=732$

$732^{2}-732-\sqrt{ } 3=-1945=1 / 514 \quad \mathrm{t}=514$ (see above)

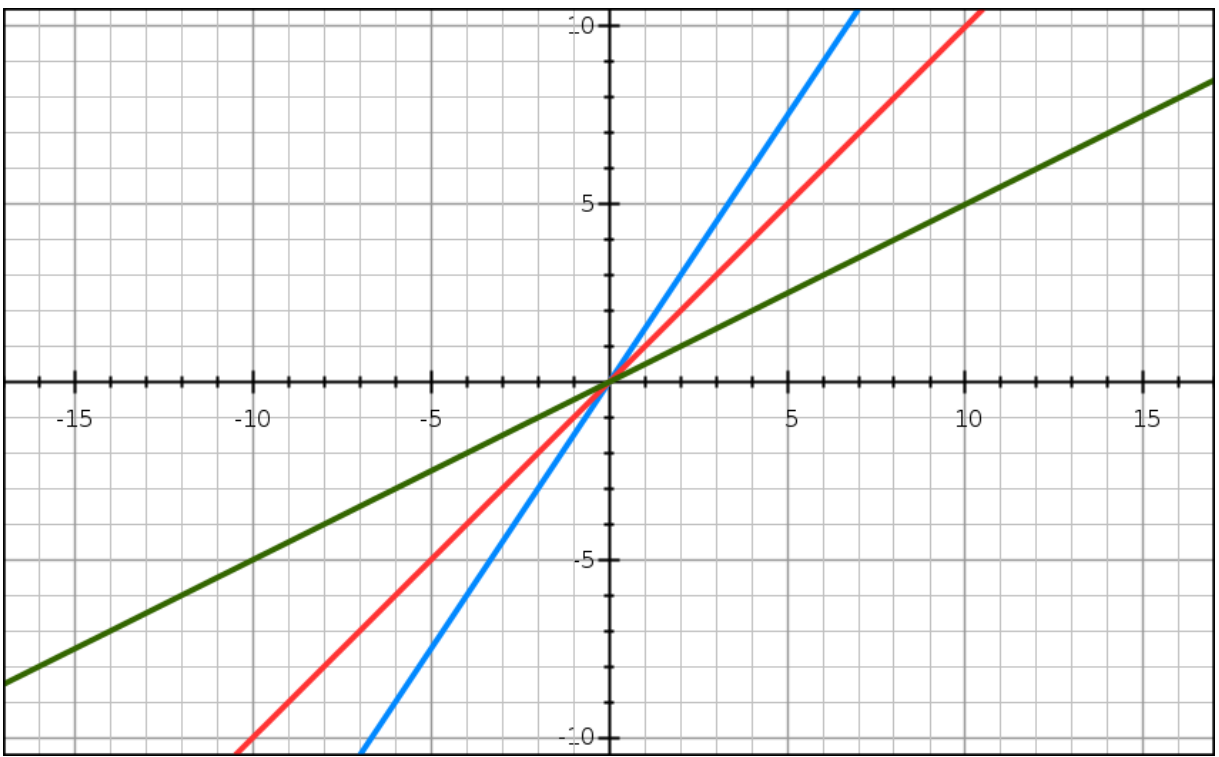

Figure1. Plot of Value Functions: Green is bad; Blue is Good; Red is neutral

\section{GAME OF LIFE PHYSICS}

There is only one rule in the Game of Life Physics. It is:

For each cell in the grid, count how many of its 8 neighbours are $O N$ at the present instant. If the answer is exactly two, that cell stays in its present state (ON or OFF) in the next instant. If the answer is exactly three, the cell is ON in the next instant whatever its current state. Under all other conditions, the cell is OFF. [1]

This translates into Chess. On =man on a cell; Off $=$ no man on a cell.

$(8$ choose 2$)=28$

$(8$ choose 3$)=56$

$56=28 \chi$

$\chi=2 \Rightarrow y=2=L \Rightarrow y=y$,

$\mathrm{SE}=2 \mathrm{t}-1$

$=2(2)-1=3$

$\mathrm{t}^{2}-\mathrm{t}-1=3$

$\mathrm{t}^{2}-\mathrm{t}-4=0$

$\mathrm{t}=256 ; 1.561$ 


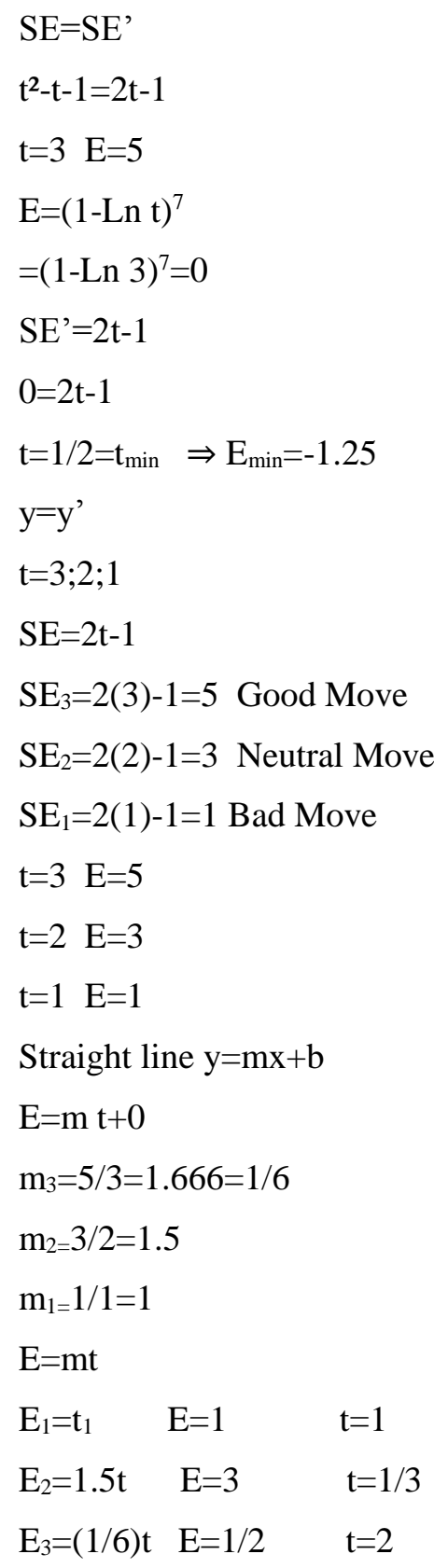

Chess invneted in India in 6th C BCE

$8 \times 8=64$ squares

Ways to move:

Pawn 2

Rook 2

Bishop 2

Kinght 4

King 1

Queen 8
8

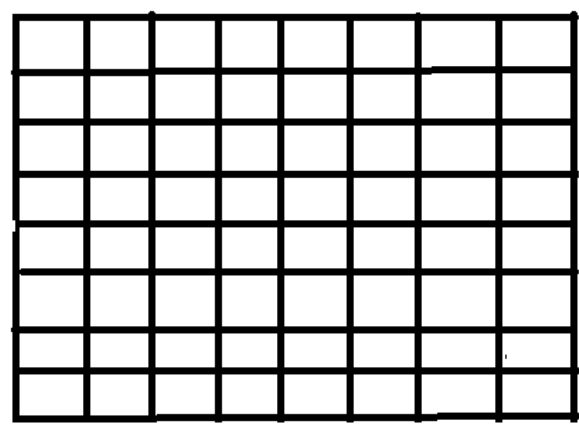

$(64$ chose 38$)=64 ! /(38 !)(28 !)$

$19 \times 2$ (Black and White) $=126.9 /(52.30)(403)$ $=38$
$62 / 6.02=$

1.0666

$\sim 105.7 \mathrm{mV}$

of the

human

nervous

system 
$\mathrm{M}=\mathrm{Ln} \mathrm{t}=\mathrm{Ln} 1 / 2=-0.693$

$\mathrm{PE}=\mathrm{Mc}^{2}+\mathrm{MGh}=0.693[9+6.67]=10.947$

$\mathrm{t}=\mathrm{KE}=1 / 2 \mathrm{Mv}^{2}=1 / 2(4)(1 / \sqrt{ } 2)^{2}=1$

$\mathrm{SE}=\mathrm{SE}^{\prime}=5$

$\mathrm{TE}=16.94 \sim 17$

$\mathrm{t}=1 / 17=590$

$\mathrm{t}^{2}-\mathrm{t}-1=1.055 \mathrm{mV}$ (Human Nervous System)

$4^{(\mathrm{n}+1)}=4^{(2669)}=404=\operatorname{Re}$

$\rho /[\mathrm{e} \times \mathrm{Re}]=127.3 /[1 \times 404]=\pi=\mathrm{t}_{\max }$.

$\{8$ choose 2$\}=28$

$\mathrm{TE}=28=6268+(\mathrm{n}+1)+\mathrm{SE}$

$6241=\mathrm{n}$

PVal. \& TLN

$\mathrm{TE}=\mathrm{PVal} \times \mathrm{TLN}$

$(8 \times 8) / 6.02=106.8=P V a l . x$ TLN

$\mathrm{M} /(2 \pi)=$ PVal $\mathrm{x}$ TLN

$\mathrm{TE}=\Sigma \mathrm{M} /(2 \pi)$

$\mathrm{TE}=3 / 2 \pi$

$\mathrm{TE}=2 / 2 \pi$

$\mathrm{TE}=1 / 2 \pi$

$\Sigma \mathrm{TE}=[3 / 2 \pi+2 / 2 \pi+1 / 2 \pi][\mathrm{PVal} . \mathrm{x}$ TLN $]$

$=3 / \pi[$ PVal. $x$ TLN $]$

Good Move $=3 / \pi($ PVal x 1$)=3$ PVal $/ \pi$

Neutral Move $=3 / \pi($ PVal x 0$)=0$

Bad Move $=3 / \pi($ PVal x $(-1)=-3 \mathrm{PVal} / \pi$

$-3 \mathrm{PVal} / \pi \leq 0 \leq 3 \mathrm{PVal} / \pi$

\section{Bad Neutral Good}

$\Sigma \mathrm{E}=3 / \pi$ [PVal. $\times \mathrm{TLN}]$

Good Move

$2=\mathrm{t}(1.5 \times 1)$

$\mathrm{t}=4 / 3$

$\mathrm{E}=3 / 4=0.75$

$\mathrm{E}=0.75(1.5 \times 1)$

$=1.125=1 / 888=\mathrm{E}$

$\mathrm{t}=0.888$

$\mathrm{t}^{2}-\mathrm{t}-1=1.098 \sim 11$

$\mathrm{E} \geq 11$

11 Dimension Universe

$2^{11} / 2^{7}=2^{4}=16 \sim 157=\pi / 2$ 
$\sim 1601=$ Charge on an electron.

Bad move $<1 / 2<$ Good move

Bad move $<1 / 2 \times 2 \pi<$ Good move

Neutral Move

$1 / 2=\mathrm{t}(\mathrm{PVal} . \mathrm{x} 0)$

$\mathrm{t}=0$

Bad Move

$0=\mathrm{t}(\mathrm{PVal} \times \mathrm{TLN})$

$0=\mathrm{t}(-1 / 2 \mathrm{x}-1)$

$0=2 \mathrm{t}$

$\mathrm{t}=0$

Game of Life Physics

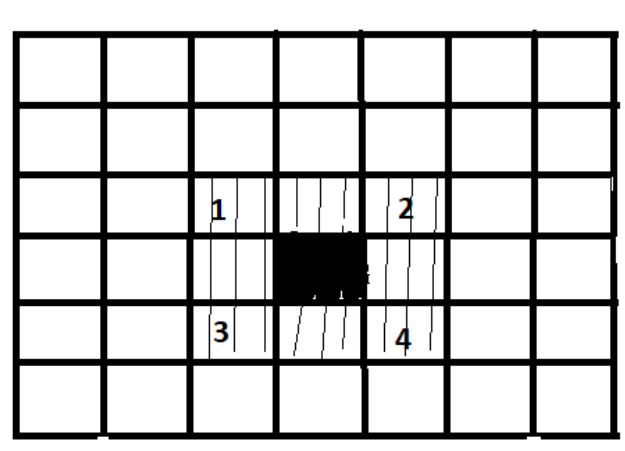

$\mathrm{TE}=\mathrm{PE}+\mathrm{KE}+\mathrm{SE}$

$=36+1+5=42=2 \times 3 \times 7$
$2 t-1=2(2)-1$

$=\mathrm{t}=3 \mathrm{E}=5$

$\mathrm{SE}=\mathrm{SE}^{\prime}$

$t=3-2=1$

$\mathrm{t}=\mathrm{KE}=1 / 2 \mathrm{Mv}^{\wedge} \mathbf{2}$

$1=1 / 2 \mathrm{Mv}^{\wedge} 2$

$2=(4) v^{\wedge} 2$

$v=1 /$ sqrt2

$\mathrm{PE}=\mathrm{Mc}^{\wedge} \mathbf{2}$

$=4(9)=36$

Figure1.

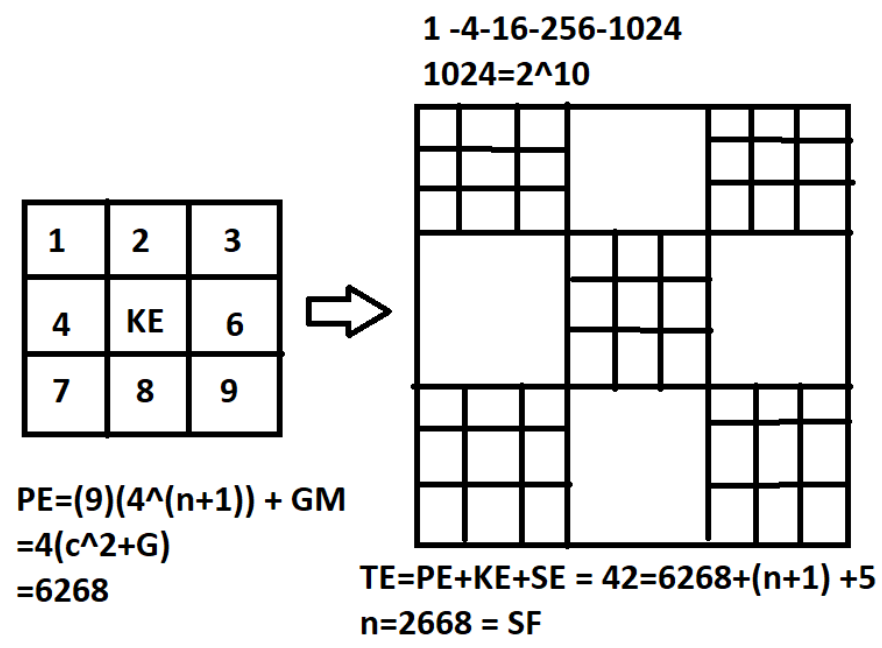

Figure2.

[ 38 choose 7] $=126.7 \times 10^{11}=\rho$

$\rho /[\mathrm{E} \times \mathrm{Re}]=126.7 /(1 \times 404)=3.12 \times 10^{9}{ }^{3}$ billion possible moves.

$3.12 / 921600 \mathrm{Bd}=56.42 \mathrm{~min}$ per move $\sim 1$ hour

International Journal of Scientific and Innovative Mathematical Research (IJSIMR) 
$\{8$ choose 2$\}=28$

$\left\{t\right.$ choose $\left.d^{2} E / d t^{2}\right\}$

$\{t$ choose $G\}$ Clairnaut DE

$\mathrm{E}=\mathrm{E}^{\prime}=\mathrm{E}^{\prime \prime}=\mathrm{G}$

$\{t$ choose $E\}$

(1/E)! /[E!(E-1/E)!

$1=28=(\mathrm{E}-1 / \mathrm{E})$

$1 / 28=(\mathrm{E}-1 / \mathrm{E})$

$1 / 28=\left[\mathrm{E}^{2}-1\right] / \mathrm{E}$

$\mathrm{E}^{2}-1=\mathrm{E} / 28$

$28\left(\mathrm{E}^{2}-\mathrm{E}-1\right)=0$

E$^{2}$-E-1=0 Golden Mean Parabola

Now,

$\{8$ choose 3$\}=$

56

$8 ! /[3 !(8-3) !=56 \quad \mathrm{t}=\sqrt{3}=$ =igenvector

$\mathrm{t}_{1} /\left[\mathrm{t}_{2}\left(\mathrm{t}_{2}-\mathrm{t}_{1}\right)=56\right.$

$\mathrm{t}_{1}=56 \mathrm{t}_{2}\left(\mathrm{t}_{2}-\mathrm{t}_{1}\right)$

$\mathrm{t}_{1}=56 \mathrm{t}_{2}-56 \mathrm{t}_{1}$

$57 \mathrm{t}_{1}=56 \mathrm{t}_{2}{ }^{2}$

$57 / 56 \mathrm{t}_{1}=\mathrm{t}_{2}{ }^{2}=(\sqrt{3})^{2}$

$\mathrm{t}_{1}=3(56) / 57=263=1 / 38=1 /(2 \times 19)$

$\mathrm{t}_{1}{ }^{2} \cdot 1 / \mathrm{t}_{1}=\{64$ choose 38$\}$

$\mathrm{t}_{1}=64 / 38=263$

$E=(1-\operatorname{Ln} t)^{7}=379$

$1 / \mathrm{E}=\mathrm{t}=263=\mathrm{t}_{1}$

$\mathrm{t}_{1 \times} \mathrm{t}_{1} /\left(1 / \mathrm{t}_{1}\right)=\{64$ choose 38$\}=56$

$\{64$ choose 38$\}=R$

$\mathrm{E}=(1-\operatorname{Ln} \mathrm{t})^{\wedge} 7$

$1.001=(1-\operatorname{Ln} \mathrm{t})$

$\mathrm{t}=1$

$\{8$ choose 2$\}=\mathrm{E}=\mathrm{t}^{2}-\mathrm{t}-1=28$

$\{8$ choose 3$\}=t=1 / E$

$\mathrm{t}=\mathrm{E}^{2}+\mathrm{E}-2$

$=28^{2}-28-2$

$=755$

$\mathrm{E}=1-\operatorname{Ln} 0.755)^{7}$

$=(1-0.6626)^{7}$

$=(1-\mathrm{h})^{7}$ 
$=1.012 \sim 1$

$\mathrm{E}=1 / \mathrm{t}$

$\{64$ choose 19$\}$

$=\mathrm{E}=(1-\operatorname{Ln} \mathrm{t})^{7}$

$=(1-\operatorname{Ln} 263) 7$

$=0.858$

$=\mathrm{R}$

\section{CONCLUSION}

AT Math and the Game of Life Physics rule with programmable Chess.

\section{REFERENCES}

[1] Dennett, DC., Darwin's Dangerous Idea., Simon and Schuster USA 1995.

Citation: PAUL T E CUSACK, Algorithm for Computer Chess, International Journal of Scientific and Innovative Mathematical Research (IJSIMR), vol. 8, no. 8, pp. 1-10, 2020. Available : DOI: https://doi.org/10.20431/2347-3142.0808001

Copyright: (C) 2020 Authors. This is an open-access article distributed under the terms of the Creative Commons Attribution License, which permits unrestricted use, distribution, and reproduction in any medium, provided the original author and source are credited. 